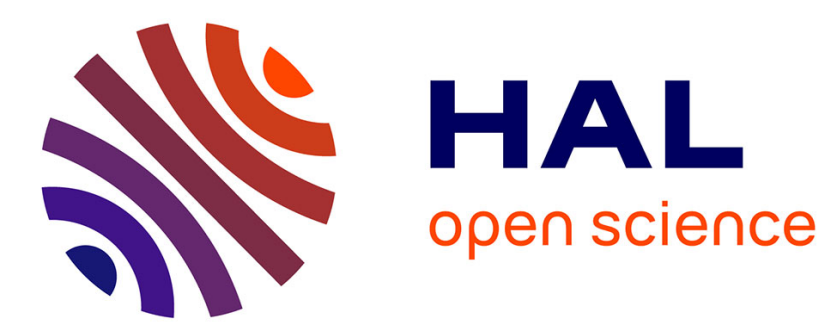

\title{
The reduced basis element method for fluid flows
}

Alf E. Lovgren, Yvon Maday, Einar M. Ronquist

\section{To cite this version:}

Alf E. Lovgren, Yvon Maday, Einar M. Ronquist. The reduced basis element method for fluid flows. 2006. hal-00112149

\author{
HAL Id: hal-00112149 \\ https://hal.science/hal-00112149
}

Preprint submitted on 10 Nov 2006

HAL is a multi-disciplinary open access archive for the deposit and dissemination of scientific research documents, whether they are published or not. The documents may come from teaching and research institutions in France or abroad, or from public or private research centers.
L'archive ouverte pluridisciplinaire HAL, est destinée au dépôt et à la diffusion de documents scientifiques de niveau recherche, publiés ou non, émanant des établissements d'enseignement et de recherche français ou étrangers, des laboratoires publics ou privés. 


\title{
The reduced basis element method for fluid flows.
}

\author{
Alf Emil Løvgren, Yvon Maday and Einar M. Rønquist
}

April 14, 2006

\begin{abstract}
The reduced basis element approximation is a discretization method for solving partial differential equations that has inherited features from the domain decomposition method and the reduced basis approximation paradigm in a similar way as the spectral element method has inherited features from domain decomposition methods and spectral approximations. We present here a review of the method directed to the application of fluid flow simulations in hierarchical geometries. We present the rational and the basics of the method together with details on the implementation. We illustrate also the rapid convergence with numerical results.
\end{abstract}




\section{Introduction}

The numerical simulations of fluid flows is a challenging task on which the computational fluid dynamic and the applied math communities have been working for years. These simulations still fill a large part of the many supercomputers of the planet in a quest for a better reproduction of real life situations. Many applications require a rapid evaluation of the flow picture corresponding to some documented natural phenomenon; among the most prominent applications in this area is the study of internal flows in hierarchical geometries as seen in medical applications. Examples include the analysis of blood flows in arteries (as e.g. Figure 1, left, which presents a reconstruction of the Willis complex used for numerical simulations [29]), and air flow in the lung (as e.g. Figure 1, right, which presents a reconstruction of the upper part of the lung used for numerical simulations [5]). A related example from engineering applications is the study of a building's infrastructure for the design of an air conditioning network.
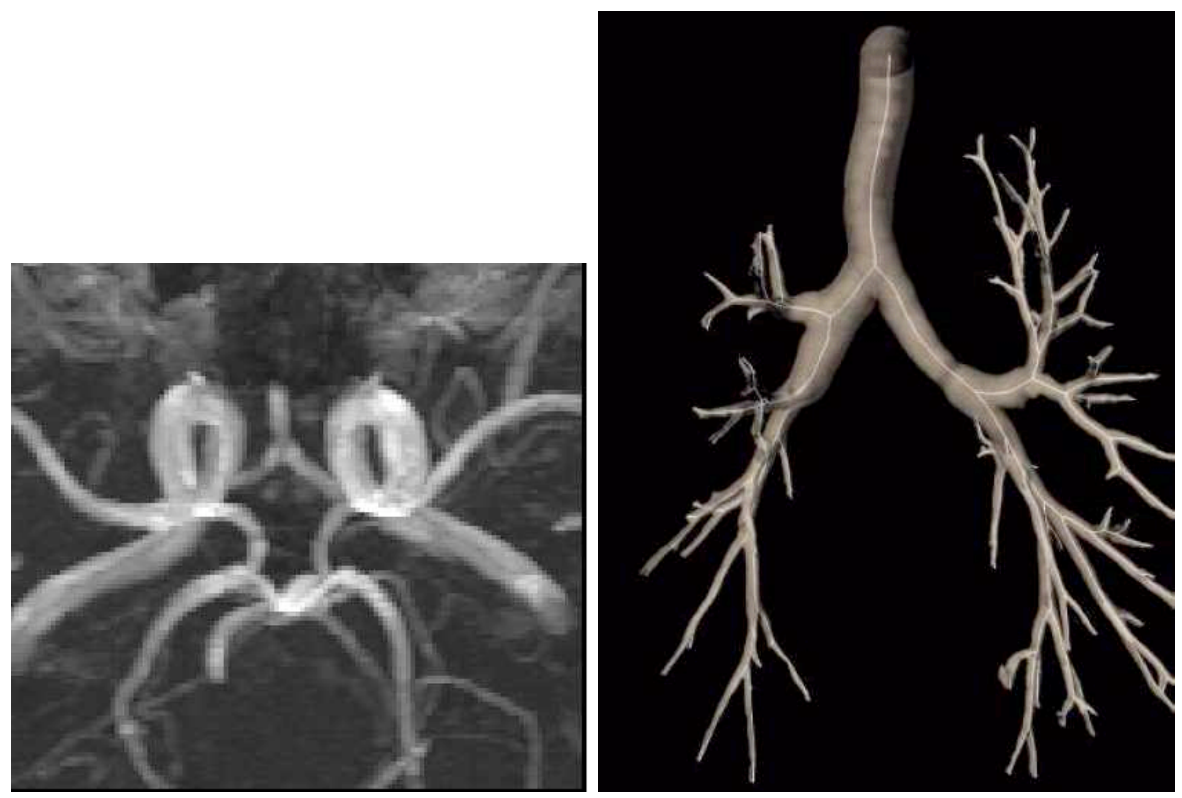

Figure 1: The left plot represents the geometry of the Willis complex that is composed of many blood vessels designing an intricate network (thanks to [29]). The right plot represents the reconstructed geometry of the upper part of the lung exhibiting a hierarchical network (thanks to [5]).

In this range of applications, the challenge of the simulations comes more from the complexity of the geometry and its representation than from the fine structures of the flow itself. Actually it can be noticed that there is some repetitiveness or similarities in the behavior of the flow that allows for the definition of reduced model strategies (see e.g. [20]). The quite general way of deriving such reduced models that will be presented in this paper combines three strategies that have received quite a lot of attention in the computational community: 
- the reduced basis method

- the domain decomposition method

- the a posteriori estimations

and has been named the reduced basis element method with rapid certificate of fidelity.

The reduced basis method is used in the case where we want to solve rapidly a large number of problems governed by some partial differential equations that depend on a parameter. The strategy is composed of two stages: the off-line computation during which a few typical problems are solved by classical discretization methods; the solutions to these problems are the basis in which the on-line computation will be performed through a Galerkin process. This approach can compete with the best high order discretization methods, and due to the off-line/on-line strategy, it is very rapid. The extreme high quality and speed of the solution procedure is balanced by the fact that this method is tuned only to particular situations. The basis is not multipurpose and should only be used in case of a parameter dependent PDE. In some sense this reduced basis method is in the spirit of "learning strategy". We refer to $[7,1,19,6,23,24,15]$.

The domain decomposition method is a "divide and conquer" approach that has benefited from the development of parallel supercomputers. The idea, when a partial differential equation on a given domain has to be solved, is to break the domain into overlapping or non overlapping subdomains and combine the solution strategies of the same PDE over the subdomains, yielding independent smaller tasks, in a proper way to iteratively approximate the solution of the global problem. This method has been very popular and has been developing rapidly over the past twenty years. We refer to e.g. [26], [30] for a general overview of the problem.

A posteriori analysis is the mathematical equivalent of "precision error bars" that are well known for real experiments and that are attached to any experimental data as a mandatory complement to any measure or output in order to know where the unreachable truth lies. Once a mathematical model is provided, in our case through the definition of the partial differential equation, complemented with the necessary initial and boundary conditions, and once the mathematical analysis allows us to specify in which sense a solution to the problem has to be sought, any numerical method aims at approximating this solution at the price of a certain amount of computation. Most often the larger the computation, the closer the approximated solution is to the "exact solution". The a posteriori analysis complements the computations with computable bounds on the approximation by quantifying the error that has been committed. We refer to $[31,8]$ for general presentations of these strategies.

In the reduced basis element method we consider the geometry of the computational domain to be the generic parameter. The domain is decomposed into smaller blocks, all of them can be viewed as the deformation of a few reference shapes. Associated with each reference shape are previously computed solutions (typically computed over different deformations of the reference shapes). The precomputed solutions are mapped from the reference shapes to the different blocks of the decomposed domain, and the solution on each block is found as a linear combination of the mapped precomputed solutions. The solutions on the different blocks are glued together using Lagrange multipliers. Our hierarchical flow systems can be decomposed into pipes and bifurcations.

In Section 2, we present the basics of the domain decomposition and the solution attached to it; actually the method can be compared with a numerical plumber toolbox 
where the elemental domain+attached basis can be hooked together. We first explain the approach on a single domain case and then on a domain decomposed geometry.

In Section 3 we present the reduced basis element approach for simulation of the Laplace, Stokes and Navier-Stokes problem.

In Section 4, we provide the basics of the numerical implementation, including a new method to generate the deformation mappings.

Finally, some numerical results are provided in Section 5 that illustrate the potential of the method together with convergence tables.

We end this introduction by indicating that the work presented here is actually motivated by the simulation of air flows in the lung. The geometry of the respiration tree is indeed of such a complexity that a multiscale/multimodel has to be constructed in order to be able to derive implementable ab initio discretization methods.

What we propose is a decomposition of this tree into 4 stages where different models will be exploited:

- the upper part, including the mouth and nose, that goes down to the first bifurcations

- the medium part, from the second or third bifurcation, down to the 8th or 10th

- the distal part, down to the acini

- the acini

and with all these stages being imbedded in a structural parenchyma.

It is then an easy matter to realize that the exact representation of the flow inside this complete tree governed by the 3D Navier Stokes equations is currently far from being achievable and will still not be for a long time. This is currently only feasible, though still quite expensive, for the upper part. It is at the level of the second part, that we refer to the reduced basis element method. Concerning the distal and the terminal part, the description of the set of acini evokes easily the reference to homogenization. This will be fractal homogenization for the former and multiphysics (fluid-structure interaction), non stationary homogenization for the latter. We refer to [4] for a first analysis in fractal homogenization and to [2] where a first fluid structure model interaction is considered.

These four different models are hooked together as is explained in [11] on a simpler model, resulting in a viable multiscale/multimodel.

\section{Basics of the reduced basis element method}

\subsection{The monodomain case}

In the reduced basis method, there is typically a parameter dependent problem to be solved for many instances of the parameter (generally denoted by $\mu$ ). In the reduced basis element method, the parameter represents the shape of the domain on which some partial differential equation has to be solved. In the single domain case, there exists a "reference domain", denoted as $\hat{\Omega}$, and the problem modeling the phenomenon of interest has to be solved on "deformations" of $\hat{\Omega}$ denoted as $\Omega_{\Phi}=\Phi(\hat{\Omega})$ where the "parameter" $\mu=\Phi$ is a regular enough, one to one, mapping.

In the reduced basis method, there is typically a fundamental assumption that the "dimension" of the set $S$ of all solutions obtained by letting the parameter take all admissible values, is small in the sense that the set of all solutions $u(\mu)$, when $\mu$ varies 
in the parameter set, can be approximated very well by its projection over a finite and low dimensional vectorial space. Then, for well enough chosen $\mu_{i}$, there exist coefficients $\alpha_{i}=\alpha_{i}^{N}(\mu)$ such that the finite sum $\sum_{i=1}^{N} \alpha_{i} u\left(\mu_{i}\right)$ is very close to $u(\mu)$ for any $\mu$. In the reduced basis element method, the parameter being the shape of the domain, analysis of the regular dependency of the solution of a PDE on the domain can be found in e.g. [21]. In addition to the theoretical analysis that may lead to believe that the assumption of the small dimension of $S$ holds qualitatively true, it is most of the time enlightening to get quantitative information on the confirmation of this fundamental assumption. In general, we suggest to perform a preliminary feasibility analysis from which we can get "experimentally" an evaluation of the $N$ width $d_{N}(S, X)$ of the set $S$ of all solutions for the different admissible parameters. Following [22], it is defined as

$$
d_{N}(S, X)=\inf _{X_{N}} \sup _{u \in S} \sup _{u_{N} \in X_{N}}\left\|u-u_{N}\right\|_{X}
$$

(where $X$ is some appropriate normed space, and $X_{N}$ is a generic finite dimensional subspace of $X$ with dimension equal to $N$ ) and it can be evaluated revealing a potential rapid convergence of this width as $N$ grows (note that we prefer the denomination "grow" to the classic one "goes to infinity" since an accuracy of $10^{-4}$ is often achieved for $N$ of order tens). This indeed will already provide a good evaluation of the potential of the reduced basis concept to work well. Note that a little bit of intuition may be useful here since it may not be the $u\left(\mu_{i}\right)$ that have a small width but some $\mathcal{F}\left[u\left(\mu_{i}\right)\right]$ or even $\mathcal{F}\left(\mu_{i}\right)\left[u\left(\mu_{i}\right)\right]$ where $\mathcal{F}$ is some simple transformation; we will see this for the Stokes problem below.

Once these basic considerations are made, the outline of the method may proceed. For some well chosen instances of the mapping, $\Phi_{1}, \Phi_{2}, \ldots, \Phi_{N}$, the solution to the problem is solved through your preferred numerical scheme (that, in our case is the spectral element method) over the domains $\Phi_{i}(\hat{\Omega})$, with a good enough accuracy. These solutions, named $u_{1}, u_{2}, . ., u_{N}$, are then stored on $\hat{\Omega}$ through an appropriate change of variables involving the mapping $\Phi_{i}: \hat{u}_{i}=\mathcal{F}_{i}\left[u_{i}\right]$. This provides (at most) $N$ functions $\hat{u}_{1}, \hat{u}_{2}, . ., \hat{u}_{N}$ over $\hat{\Omega}$, linearly independent, selected in the set $\hat{S}$ of all possible solutions mapped back onto $\hat{\Omega}$. An example of such an appropriate change of variables is $\hat{u}_{i}=u_{i} \circ \Phi_{i}$, but we will see that more involved changes of variables may be proposed. For any new problem to be solved over the domain $\Omega_{\Phi}=\Phi(\hat{\Omega})$ characterized by the data of the transformation $\Phi$, an approximation of the corresponding solution $u_{\Phi}$ is sought in the vectorial space spanned by the $\tilde{u}_{1}, \tilde{u}_{2}, . ., \tilde{u}_{N}$, where the $\tilde{u}_{i}$ are functions defined over $\Omega_{\Phi}$ from the $\hat{u}_{i}$ through the same appropriate change of variables involving now the mapping $\Phi$. In order for the approximated solution to be, up to a constant, as good as the analysis of the width might indicate, a Galerkin process is generally used, since from Céa's lemma, the error between the exact solution and the numerical one is upper bounded by some constant times the best fit error.

\subsection{The multidomain case: the plumber's toolbox}

Let us now add the domain decomposition argument. First, we assume that the domain $\Omega$ where the computation should be performed can be written as the non-overlapping union of subdomains $\Omega^{k}$ :

$$
\bar{\Omega}=\bigcup_{k=1}^{K} \bar{\Omega}^{k}, \quad \Omega^{k} \cap \Omega^{\ell}=\emptyset, \text { for } k \neq \ell .
$$


Next, we assume that, as was said in the monodomain case, each subdomain $\Omega^{k}$ is the deformation of the "reference" domain $\hat{\Omega}$ through a regular enough, and one to one, mapping. Together with this geometric decomposition a functional decomposition is proposed since every $\Omega^{k}$ actually comes filled with the basis functions derived from the $\hat{u}_{1}, \hat{u}_{2}, . ., \hat{u}_{N}$. This allows us to define the finite dimensional space

$$
Y_{N}=\left\{v \in L^{2}(\Omega), v_{\mid \Omega^{k}}=\sum_{i=1}^{N} \alpha_{i}^{k} \mathcal{F}_{k}^{-1}\left[\hat{u}_{i}\right]\right\}
$$

which is a set of uncoupled, element by element, discrete functions. This is generally not yet adequate for the approximation of the problem of interest since some glue at the interfaces $\gamma_{k, \ell}$ between two adjacent domains $\bar{\Omega}^{k} \cap \bar{\Omega}^{\ell}$ has to be added to the elements of $Y_{N}$, the glue depending on the type of equations we are interested to solve (it will be relaxed $C^{0}$-continuity condition for a Laplace operator, or more generally relaxed $C^{1}$-continuity condition for a fourth-order operator ${ }^{1}$ ).

At this stage it should be noticed that, modulo an increase of complexity in the notations, there may exist not only one reference domain $\hat{\Omega}$ filled with its reduced basis functions but a few numbers so that the user can have more flexibilities in the design of the final global shape by assembling deformed basic shapes like a plumber would do for a central heating installation.

The reduced basis element method is then defined as a Galerkin approximation over the space $X_{N}$ being defined from $Y_{N}$ by imposing these relaxed continuity constraints. We refer to the next section for details concerning the way the relaxed continuity conditions are imposed.

\section{The reduced basis element method in action}

\subsection{The Laplace problem}

We synthesize here the experiments that have been done on the Laplace problem. The motivation comes from the design of an optimal thermal fin. Typical $K=3$-stages and $K=4$-stages thermal fins we consider are depicted and described in Figure 2.

We assume no heat generation within the thermal fin itself. A constant heat flux, $q$ (generated from an electronic device, say) enters the fin at the fin root, $\Gamma_{\text {root }}$, and leaves through the remaining surface of the fin. One motivation for this analysis can be to optimize the fin (the number of stages, the physical dimensions, and the thermal conductivities) so as to best remove this heat.

The heat loss from the fin surface due to convection, is modeled by prescribing Robin type boundary conditions

$$
-\kappa \frac{\partial u}{\partial n}=\operatorname{Bi} u \quad \text { on } \quad \partial \Omega \backslash \Gamma_{\text {root }},
$$

where $\kappa$ denotes the piecewise constant conductivity in $\Omega$.

The governing equation for the temperature $u$ in the fin is the Laplace equation; more precisely, the thermal fin problem can be stated in variational form as :

Find $u \in H^{1}(\Omega)$ such that

$$
a_{\Omega}(u, v)=f_{\Omega}(v) \quad \forall v \in H^{1}(\Omega),
$$

\footnotetext{
${ }^{1} \mathrm{~A}$ precise definition of the meaning of relaxed is proposed in the next section
} 

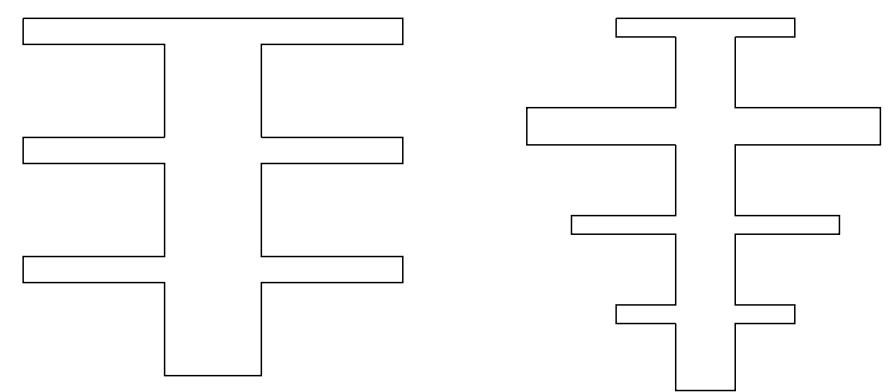

Figure 2: The left plot shows a typical $K=3$-stages fin with similar wings, the right one shows a symmetric $K=4$-stages fin with a variable wing-size. The root of the fin is at the bottom.

where

$$
\begin{aligned}
a_{\Omega}(u, v) & =\int_{\Omega} \kappa \nabla u \cdot \nabla v d A+\mathrm{Bi} \int_{\partial \Omega \backslash \Gamma_{\text {root }}} u v d S \\
f_{\Omega}(v) & =\int_{\Gamma_{\text {root }}} q v d S,
\end{aligned}
$$

where we remind that $\kappa$ is piecewise constant and is a (multi)parameter to be optimized. We note that $a_{\Omega}(\cdot, \cdot)$ is a symmetric, positive definite bilinear form, and $f_{\Omega}(\cdot)$ is a linear form. It is standard to show that this problem has a unique solution $u$.

When solving the problem on various fins, we observe that the temperature distribution is characterized by a certain amount of "repetitiveness" over the stages of the fin. This has led us to propose to view each stage of the fin as the deformation of a reference domain; hence we write

$$
\bar{\Omega}=\cup_{k=1}^{K} \overline{\Omega^{k}}
$$

where each "building block" $\Omega^{k}$ is assumed to be the image of a reference one-stage fin $\hat{\Omega}$. The mapping $\Phi_{k}$ between $\hat{\Omega}$ and $\Omega^{k}$ is here chosen piecewise linear (and obviously continuous). We illustrate in Figure 3 the choice of reference domain $\hat{\Omega}$. The reduced basis element method assumes that $\hat{\Omega}$ is provided with basis functions $\hat{\zeta}_{1}, \hat{\zeta}_{2}, \ldots, \hat{\zeta}_{N}$, that are supposed to be linearly independent and mapped over each $\Omega^{k}$ through $\Phi_{k}$. We thus introduce the space

$$
Y_{N}(\Omega)=\left\{v_{M} \in L^{2}(\Omega) \mid \quad v_{M \mid \Omega^{k}} \circ \Phi_{k} \in \operatorname{span}\left\{\hat{\zeta}_{1}, \hat{\zeta}_{2}, \ldots, \hat{\zeta}_{N}\right\}\right\}
$$




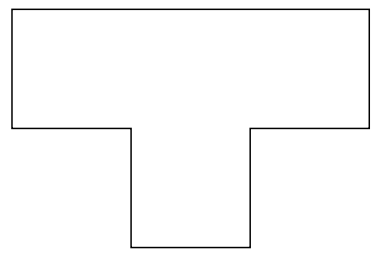

Figure 3: the reference stage $T$-shape domain.

Note again that $Y_{N}(\Omega)$ is not an acceptable discretization space for $H^{1}(\Omega)$, which leads us to define a subspace $X_{N}(\Omega)$ by gluing the functions of $Y_{N}(\Omega)$ across the interfaces $\gamma_{k, \ell}$ between two adjacent stages

$$
\bar{\gamma}_{k, \ell}=\overline{\Omega^{k}} \cap \overline{\Omega^{\ell}} .
$$

Like in the mortar element method, the matching, expressing this "relaxed continuity" is done in a variational way by imposing

$$
X_{N}(\Omega)=\left\{v_{N} \in Y_{N}(\Omega) \mid \quad \int_{\gamma_{k, \ell}}\left(v_{N \mid \Omega^{k}}-v_{N \mid \Omega^{\ell}}\right) q=0, \quad \forall q \in W_{k, \ell}\right\},
$$

where the space $W_{k, \ell}$ is defined in a proper way. An easy choice is the set of all polynomials on the interface $\mathbb{P}_{n}\left(\gamma_{k, \ell}\right)$ with degree $\leq n$ but a smarter choice, based on the numerical analysis of nonconforming approximations, is to span $W_{k, \ell}$ with a few selected normal derivatives of the solutions that have been precomputed to construct the basis functions $\hat{\zeta}_{1}, \hat{\zeta}_{2}, \ldots, \hat{\zeta}_{N}$.

The discrete problem then reads : Find $u_{N}$ in $X_{N}(\Omega)$ such that

$$
\underline{\mathrm{a}}_{\Omega}\left(u_{N}, v_{N}\right)+\operatorname{Bi} \int_{\partial \Omega \backslash \Gamma_{\mathrm{root}}} u v d S=f_{\Omega}\left(v_{N}\right), \quad \forall v_{N} \in X_{N}(\Omega)
$$

where we have introduced the notation $\underline{\mathrm{a}}_{\Omega}\left(u_{N}, v_{N}\right)=\sum_{k} \int_{\Omega^{k}} \kappa \nabla u \cdot \nabla v d A$ since the functions $u_{N}$ are not in $H^{1}(\Omega)$ any more. It is standard to state that there exists a unique solution $u_{N}$ to this discrete problem and that there is a constant $C>0$, that is a function of the geometry of the problem, such that

$$
\left\|u-u_{N}\right\|_{H^{1}(\Omega)} \leq C \inf _{v_{N} \in X_{N}(\Omega)}\left\|u-v_{N}\right\|_{H^{1}(\Omega)}+\text { consistency error. }
$$


In the absence of a general theory about the status of the best fit that appears on the right hand side (see however [16]), the feasibility experiment that we quoted in subsection 2.1 allows us to get an idea of the size of the best fit. The consistency error involves the best fit of the fluxes at the interface $\gamma_{k, \ell}$ by elements of $W_{k, \ell}$.

Before closing this subsection, we should explain how the basis functions are actually chosen. As we have seen, these basis functions are to be used over varying fins with a different number of stages. The computations (here using the spectral element method, but this is not so important) have thus been done on a series of two-stage fins with various dimensions and conductivities. The corresponding solutions then give two candidates for a reduced basis over a single-stage geometry by domain reduction. After a simple change of variables, these solutions provide functions defined over the reference geometry $\hat{\Omega}$ and the basis functions $\hat{\zeta}_{1}, \hat{\zeta}_{2}, \ldots, \hat{\zeta}_{N}$ are selected within an ensemble of such functions (we make precise a selection procedure in subsection 4.1).

Remark It should be noticed here that the reduced basis element method, applied to the fin problem, has a lot a similarity with the plain reduced basis method that has been extensively used on this example for illustrating the power of the method (see $[17],[25])$. However, note that there is an additional dimension to the reduced basis element method due to the possibility of varying the number of stages. Even more, no precomputation (using your preferred method) on a four-stage problem has ever to be done to use the reduced element method. The precomputations are done on a two-stage fin, and the reduced basis element method can be applied on a fin with any number of stages.

\subsection{The steady Stokes problem}

A typical example of a hierarchical flow system $\Omega$ for which we are interested in simulating is shown in Figure 4 left. It has an inflow boundary $\Gamma_{i n}$, an outflow boundary $\Gamma_{\text {out }}$, and wall boundaries $\Gamma_{w}$. As in the previous subsection this type of domain is composed of a non overlapping union of pipes (being obtained by deformations of a reference pipe $\hat{\mathcal{P}}=(-1,1)^{2}$ as illustrated in Figure 5) and bifurcations (being obtained by deformations of the reference bifurcation $\hat{\mathcal{B}}$, represented in Figure 4 right, as is illustrated in Figure 6, see also Figure 7).

In what follows we thus assume that

$$
\bar{\Omega}=\bigcup_{k=1}^{K} \bar{\Omega}^{k}, \text { where each } \Omega^{k}=\Phi^{k}(\hat{\Lambda}),
$$

where $\Lambda$ stands for $\mathcal{P}$ or $\mathcal{B}$ and $\Phi^{k}$ is a sufficiently regular and one to one mapping.

On such domains we introduce the velocity space

$$
X(\Omega)=\left\{\boldsymbol{v} \in\left(H^{1}(\Omega)\right)^{2}, \boldsymbol{v}_{\mid \Gamma_{w}}=0, v_{t \mid \Gamma_{\text {in }}}=v_{t \mid \Gamma_{\text {out }}}=0\right\},
$$

where $v_{t}$ is the tangential velocity component. We also define a pressure space

$$
M(\Omega)=L^{2}(\Omega) .
$$

We assume that the flow is governed by the two-dimensional steady Stokes equations that, again written in variational formulation reads: find the velocity $\boldsymbol{u}=$ $\left(u_{1}, u_{2}\right) \in X(\Omega)$ and the pressure $p \in M(\Omega)$ such that

$$
\begin{array}{lccc}
a_{\Omega}(\boldsymbol{u}, \boldsymbol{v})+b_{\Omega}(\boldsymbol{v}, p) & = & l_{\Omega}(\boldsymbol{v}) & \forall \boldsymbol{v} \in X(\Omega) \\
b_{\Omega}(\boldsymbol{u}, q) & = & 0 & \forall q \in M(\Omega),
\end{array}
$$



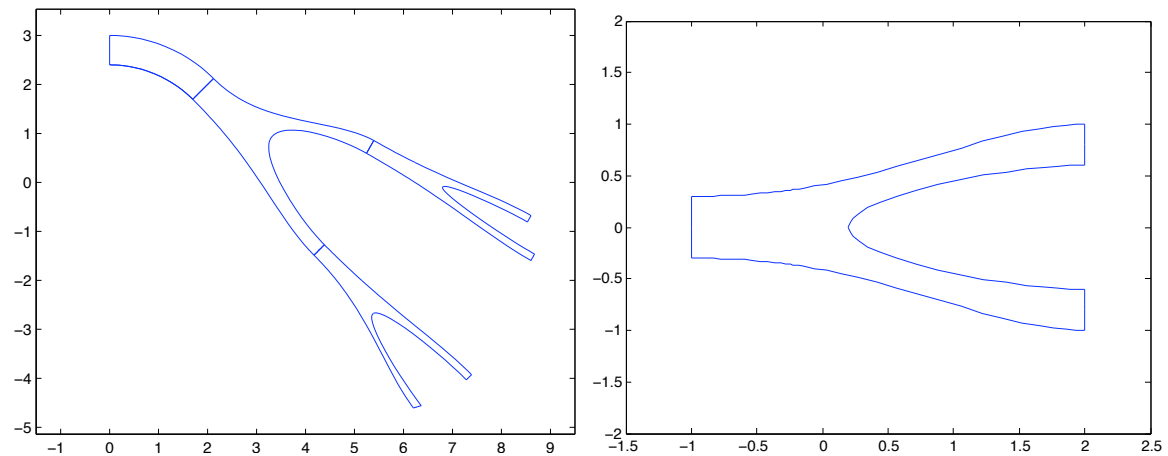

Figure 4: The left plot shows a typical domain for our flow problem. The inflow boundary is on the left and the 4 outflow boundaries are on the right. The domain has one pipe-block and three bifurcation-blocks. The plot on the right displays our reference bifurcation $\hat{\mathcal{B}}$.

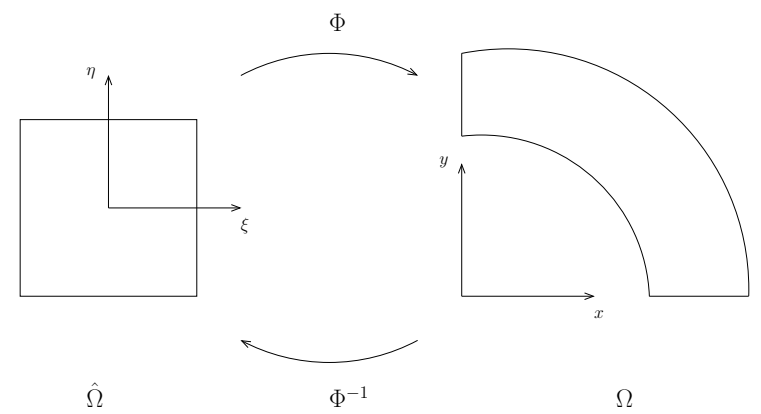

Figure 5: Mapping of the reference domain.

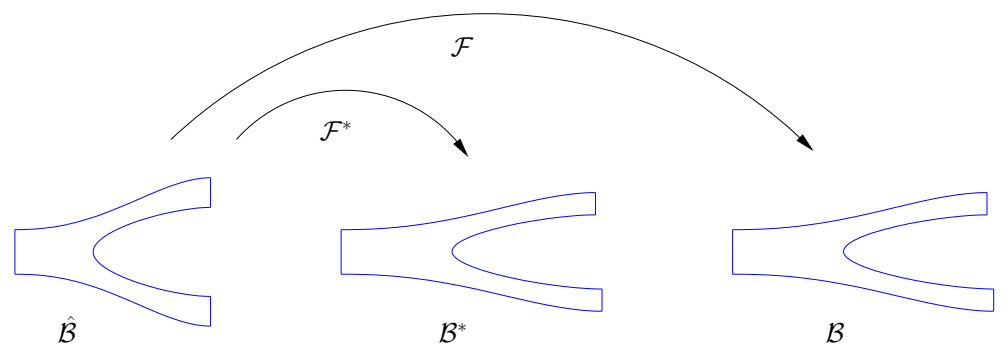

Figure 6: Different mappings for the bifurcation domains. 

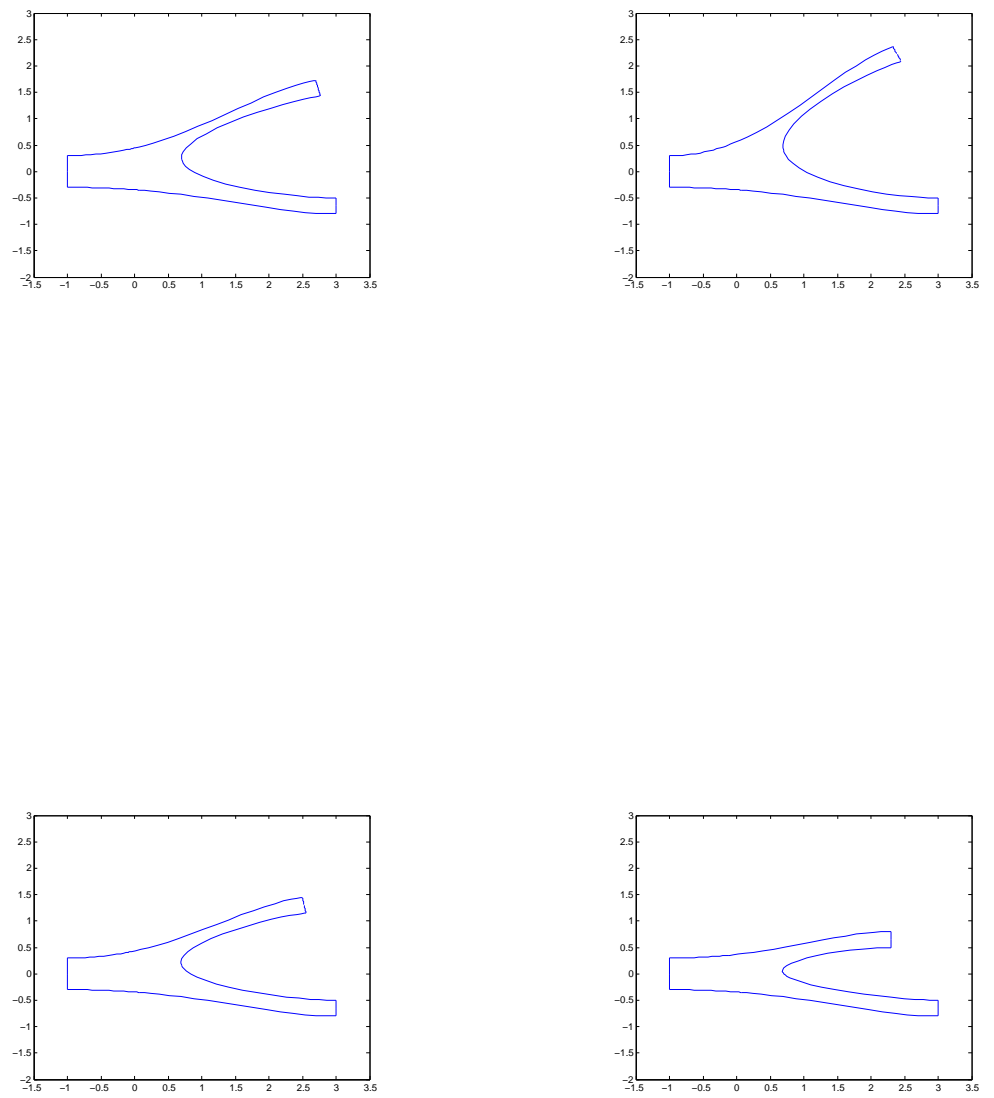

Figure 7: A few examples of deformations that are used to build the reduced basis on bifurcations. 
where

$$
\begin{aligned}
a_{\Omega}(\boldsymbol{v}, \boldsymbol{w}) & =\int_{\Omega} \nabla \boldsymbol{v} \cdot \nabla \boldsymbol{w} d A, \\
b_{\Omega}(\boldsymbol{v}, q) & =-\int_{\Omega} q \nabla \cdot \boldsymbol{v} d A,
\end{aligned}
$$

and

$$
l_{\Omega}(\boldsymbol{v})=\int_{\Gamma_{\text {in }}} \sigma_{n}^{\text {in }} \boldsymbol{v} \cdot \mathbf{n} d S+\int_{\Gamma_{\text {out }}} \sigma_{n}^{\text {out }} \boldsymbol{v} \cdot \mathbf{n} d S .
$$

The right hand side means that, in addition to the homogeneous Dirichlet boundary conditions, we have the Neumann type boundary conditions given by specifying $\sigma_{n}=$ $\frac{\partial u_{n}}{\partial n}-p$ to be $\sigma_{n}^{i n}=-1$ along $\Gamma_{i n}$ and $\sigma_{n}^{\text {out }}=0$ along $\Gamma_{\text {out }}$; here, $u_{n}$ is the normal velocity component and $\partial / \partial n$ denotes the derivative in the outward normal direction.

We now define the reduced basis solution spaces: $X_{N}(\Omega)$, which will be an approximation of $X(\Omega)$, and $M_{N}(\Omega) \subset M(\Omega)$.

Similar to what was done for the Laplace problem, we want to "fill" the reference blocks $\hat{\mathcal{P}}$ (resp. $\hat{\mathcal{B}}$ ) with basis functions $\hat{\boldsymbol{u}}_{i}$ and $\hat{p}_{i}$ that will come from preliminary solutions of the Stokes problem (computed again with your preferred method) over small unions of deformations of pipes and bifurcations. These basis functions will be obtained by first truncating the Stokes solutions, composed of a velocity vector field and a pressure, over one deformed pipe (resp. one deformed bifurcation). The results of these truncations will then be mapped onto the the reference pipe $\hat{\mathcal{P}}$ (resp. the reference bifurcation $\hat{\mathcal{B}}$ ) through an appropriate change of variables. The change of variable for the pressure basis is the same as what was done for the temperature, in the previous subsection. Mapping the velocity is somehow more subtle. Indeed, we first note that the velocities are relatively parallel to the Dirichlet boundaries; we would like to maintain this property through the mapping, second, the velocities are divergence free vector fields on the deformed domain $\Lambda=\Phi(\hat{\Lambda})$, with again $\Lambda=\mathcal{P}$ or $\mathcal{B}$, and we would like the velocity basis to keep this property when mapped to the reference domain. The Piola transformation (see [27] and [3] for general properties) allows for this constraint:

$$
\hat{\boldsymbol{u}}=\Psi(\boldsymbol{u})=\mathcal{J}^{-1}(\boldsymbol{u} \circ \Phi)|J|,
$$

where $\mathcal{J}$ is the Jacobian matrix of $\Phi$ and $|J|$ its determinant. Let us insist on the fact that, would we map the velocities as scalar functions, the reduced basis method might work nevertheless, but the convergence rate would certainly not be as good as if we transform the velocities through the Piola transformation. Actually, it is well known that a divergence free field is, in $2 \mathrm{D}$ at least, the curl of a unique potential $\Psi$. The Piola transformation appears to be associated with the simplest mapping expressed on this potential, which is certainly a natural idea. In the reduced basis context, being smart pays off.

This allows us to define the reduced basis reference spaces as

$$
\begin{aligned}
M_{N}(\hat{\Lambda}) & =\operatorname{span}\left\{\hat{p}_{i}, \quad i=1, \ldots, N\right\} . \\
V_{N}(\hat{\Lambda}) & =\operatorname{span}\left\{\hat{\boldsymbol{u}}_{i}, \quad i=1, \ldots, N\right\},
\end{aligned}
$$

Note here that the basis functions $\hat{\boldsymbol{u}}_{i}$ are divergence free (thus the standard notation " $V$ " for the associated space, see e.g. [9]).

The definition of the global spaces over $\Omega$ then proceeds from (11) by first setting

$$
M_{N}(\Omega)=\left\{p \in L^{2}(\Omega), p_{\mid \Omega^{k}}=\hat{p} \circ\left(\Phi_{k}\right)^{-1}, \hat{p} \in M_{N}(\hat{\Lambda})\right\},
$$


and then define the velocity space to be

$V_{N}(\Omega)=\left\{\boldsymbol{u} \in L^{2}(\Omega)^{2}, \boldsymbol{u}_{\mid \Omega^{k}}=\Psi^{-1}(\hat{\boldsymbol{u}}), \hat{\boldsymbol{u}} \in V_{N}(\hat{\Lambda}), \int_{\gamma_{k, \ell}}\left(\boldsymbol{u}_{\mid \Omega^{k}}-\boldsymbol{u}_{\mid \Omega^{\ell}}\right) q=0, \quad \forall q \in W_{k, \ell}\right\}$.

Here $\gamma_{k, \ell}$ again denotes the interface between two adjacent subdomains $\Omega^{k}$ and $\Omega^{\ell}$ and $W_{k, \ell}$ is a space of (vectorial) gluing functions.

It is interesting to note that, aside from the discontinuity across the interfaces, these discrete functions are perfect for the approximation of the velocity over $\Omega$ since they are divergence free. The approximation of the velocity solution of (14) can be obtained by solving only the "Laplace like" problem: find $\boldsymbol{u}_{N} \in V_{N}(\Omega)$ such that

$$
\underline{\mathrm{a}}_{\Omega}\left(\boldsymbol{u}_{N}, \boldsymbol{v}_{N}\right)=l_{\Omega}\left(\boldsymbol{v}_{N}\right) \quad \forall \boldsymbol{v}_{N} \in V_{N}(\Omega),
$$

where we remind that the notation $\underline{\mathrm{a}}_{\Omega}$ refers to the fact that the integral over $\Omega$ is split into a sum of integrals over $\Omega^{k}$. In order to recover an approximation of the pressure, we proposed in [13] to first solve the problems: find $\hat{\boldsymbol{v}}_{i}=\arg \max _{\boldsymbol{w} \in\left(H_{0}^{1}(\hat{\Omega})\right)^{2}} \frac{\int_{\hat{\Omega}} \hat{p}_{i} \nabla \cdot \boldsymbol{w}}{\left(\int_{\hat{\Omega}} \nabla \boldsymbol{w}^{2}\right)^{1 / 2}}$, then define the reference space

$$
Z_{N}(\hat{\Lambda})=\operatorname{span}\left\{\hat{\boldsymbol{v}}_{i}, i=1, \ldots, N\right\}
$$

and the global space

$$
Z_{N}(\Omega)=\left\{\boldsymbol{v} \in L^{2}(\Omega)^{2}, \boldsymbol{v}_{\mid \Omega^{k}}=\Psi^{-1}(\hat{\boldsymbol{v}}), \hat{\boldsymbol{v}} \in Z_{N}(\hat{\Lambda})\right\}
$$

where we note that no interface condition should be imposed since these are locally $H_{0}^{1}$ functions. It is an easy matter to check that the inf sup condition is satisfied on the pair $Z_{N} \times M_{N}$

$$
\inf _{q \in M_{N}} \sup _{\boldsymbol{v} \in Z_{N}} \frac{\underline{\mathrm{b}}_{\Omega}(\boldsymbol{v}, q)}{\|\boldsymbol{v}\|_{H^{1}}}=\beta,
$$

(again the notation $\underline{\mathrm{b}}_{\Omega}$ refers to the fact that the integral over $\Omega$ is split into a sum of integrals over $\Omega^{k}$ ) where $\beta>0$ may depend on $N$ and $\Omega$. This allows for recovering the discrete pressure by solving

$$
\underline{\mathrm{b}}_{\Omega}\left(p_{N}, \boldsymbol{v}_{N}\right)=l_{\Omega}(\boldsymbol{v})-\underline{\mathrm{a}}_{\Omega}\left(\boldsymbol{u}_{N}, \boldsymbol{v}_{N}\right), \quad \forall \boldsymbol{v}_{N} \in Z_{N} .
$$

Note that by setting $X_{N}=V_{N} \oplus Z_{N}$ we have solved the Galerkin approximation of (14): find $\boldsymbol{u}_{N} \in X_{N}$ and $p_{N} \in M_{N}$ such that

$$
\begin{aligned}
& \underline{\mathrm{a}}_{\Omega}\left(\boldsymbol{u}_{N}, \boldsymbol{v}_{N}\right)+\underline{\mathrm{b}}_{\Omega}\left(\boldsymbol{v}_{N}, p_{N}\right)=l_{\Omega}\left(\boldsymbol{v}_{N}\right) \quad \forall \boldsymbol{v}_{N} \in X_{N}(\Omega), \\
& \underline{\mathrm{b}}_{\Omega}\left(\boldsymbol{u}_{N}, q_{N}\right) \quad=\quad 0 \quad \forall q_{N} \in M_{N}(\Omega) \text {, }
\end{aligned}
$$

Again, standard arguments in numerical analysis allow us to state that

$$
\left\|\boldsymbol{u}-\boldsymbol{u}_{N}\right\|_{H^{1}} \leq C \inf _{\boldsymbol{v}_{N} \in V_{N}}\left\|\boldsymbol{u}-\boldsymbol{v}_{N}\right\|_{H^{1}}+\text { consistency error, }
$$

where an initial feasibility experiment may reveal how fast it goes to zero. Note that, due to the fact that the functions in the discrete space $V_{N}$ are divergence free, the behavior of the inf sup parameter $\beta$ with respect to $N$ does not appear in this estimate. However, it does appear in the pressure approximation. 


\subsection{The steady Navier-Stokes problem}

The extension to the steady Navier Stokes equation is straightforward, at least as long as we stay at the level of the definition of the discrete problem and the numerical analysis. However, the implementation involves additional difficulties that we shall treat in the next section. First, let us recall a possible variational formulation of the problem: find the velocity $\boldsymbol{u}=\left(u_{1}, u_{2}\right) \in X(\Omega)$ and the pressure $p \in M(\Omega)$ such that

$$
\begin{aligned}
& a_{\Omega}(\boldsymbol{u}, \boldsymbol{v})+b_{\Omega}(\boldsymbol{v}, p)+c(\boldsymbol{u}, \boldsymbol{v} ; \boldsymbol{u})=l_{\Omega}(\boldsymbol{v}) \quad \forall \boldsymbol{v} \in X(\Omega), \\
& b_{\Omega}(\boldsymbol{u}, q) \quad=\quad 0 \quad \forall q \in M(\Omega) \text {, }
\end{aligned}
$$

where the nonlinear term $c$ take into account the convection contribution in the equations. This one is chosen here to be written as

$$
2 c(\boldsymbol{u}, \boldsymbol{v} ; \boldsymbol{w})=\int_{\Omega} \boldsymbol{w} \cdot \nabla \boldsymbol{u} \boldsymbol{v} d A-\int_{\Omega} \boldsymbol{w} \cdot \nabla \boldsymbol{v} \boldsymbol{u} d A
$$

to maintain stability of the discretization. Note that the inflow and outflow boundary conditions involves now the dynamical pressure $p+\frac{u^{2}}{2}$ instead of the pressure $p$.

The discretization space is again built from the computation of snapshots of the Navier-Stokes equations over the union of a few deformed references domains. These solutions are then restricted to one subdomain to provide, after the proper mapping, elements in $V_{N}(\hat{\Lambda})$ and $M_{N}(\hat{\Lambda})$. Finally, for a new instantiation of the geometry, the spaces $V_{N}(\Omega), M_{N}(\Omega), Z_{N}(\Omega)$ and $X_{N}(\Omega)$ are defined as in the linear situation.

Under standard hypothesis on the solution we are interested in for the Navier Stokes problem, a convergence proof similar to (27) can again be obtained.

\section{Numerical implementation}

We start by emphasizing that that any reduced basis method necessarily involves the implementation of a more "classical" approximation method. Indeed - except for very particular and uninteresting problems - the knowledge of the solutions, that we named $u_{i}$, is impossible without referring to a discretization method (e.g. of finite element, spectral type...). This has some implications.

First of all, as explained in detail in [13], this blurs the statements on the reduced element method for the Stokes problem since the discrete reduced basis velocity functions are then not exactly divergence free any more. However, the divergence is very small, and is related to the discretization error. We have preferred to hide the difficulties that this involves since these are mostly technical issues, but when you want to be "less platonic" about the method and really try to implement it, you do have to deal with these technicalities.

The second difficulty is more general and comes from the fact that the solutions are only known through a preliminary basis, which, if we want the solution $u_{i}$ to be well approximated, has to be very large. Knowing this, the rule of the game for the efficient implementation of any reduced basis method is to strictly prohibit any online reference to the extended basis. We allow offline precomputations of the solutions (that involves the extended basis) and some offline cross contribution of these solutions (based on their expression with respect to the extended basis) but this is forbidden online. We explain in the next section how this can be done. 


\subsection{Black box approach}

From what we have just seen, both the Laplace problem and - with different notations - the Stokes problem, take the following form in the single domain case: find $u_{N} \in$ $X_{N}(\Omega)$

$$
a_{\Omega}\left(u_{N}, v_{N}\right)=f_{\Omega}\left(v_{N}\right), \quad \forall v_{N} \in X_{N} .
$$

The solution $u_{N}$ is sought as a linear combination of $\left\{u_{j}\right\}_{j=1, \ldots, \operatorname{dim} X_{N}}$ defined over $\Omega$, locally, by a proper mapping of the $\left\{\hat{u}_{i}\right\}_{i=1, N}$ stored on a reference domain $\hat{\Lambda}$. First of all, it should be indicated that it is safer to define - through a GramSchmidt orthonormalization process - an orthonormal basis $\left\{\hat{\zeta}_{i}\right\}_{i=1, N}$ spanning the same space as the $\left\{\hat{u}_{i}\right\}_{i=1, N}$, that allows us to define $\zeta_{j}$ 's over $\Omega$ by mapping and gluing as is explained in subsection 2.2. This does not change the potential approximation properties of the reduced basis, but improves, to a large extent, the stability of the implementation. The solution procedure involves the evaluation of the elements of the stiffness matrix $a_{\Omega}\left(\zeta_{i}, \zeta_{j}\right)$. This computation involves some necessary differentiation and the evaluation of integrals over $\Omega$, and this may be very costly. It should be stated here that the implementation of the reduced type method has to be much faster than the solution procedure that was used to compute the reduced basis, where much means many orders of magnitude. The $\mathcal{O}\left(\left(\operatorname{dim} X_{N}\right)^{2}\right)$ entries of the stiffness matrix have thus to be evaluated through some smart way.

Let us begin by the easy case that is named affine parametric dependence where the entries $a_{\Omega}\left(\zeta_{i}, \zeta_{j}\right)$ appear to read

$$
a_{\Omega}\left(\zeta_{i}, \zeta_{j}\right)=\sum_{p} g_{p}(\Omega) a_{p}\left(\hat{\zeta}_{n}, \hat{\zeta}_{m}\right)
$$

where the bilinear forms $a_{p}$ are domain independent. This is the case for the fin geometry where each subdomain, corresponding to one fin-stage, is composed of 4 rectangles that all map to a square through a simple affine mapping. Each $a_{p}$ is the integral over a square of $\frac{\partial \hat{\zeta}_{n}}{\partial x} \frac{\partial \hat{\zeta}_{m}}{\partial x}$ or $\frac{\partial \hat{\zeta}_{n}}{\partial y} \frac{\partial \hat{\zeta}_{m}}{\partial y}$ while the $g_{p}(\Omega)$ take into account the dimension of the corresponding rectangle and the conductivity that, due to our hypothesis, is constant.

The expensive computation of the $a_{p, n, m}=a_{p}\left(\hat{\zeta}_{n}, \hat{\zeta}_{m}\right)$ can be done offline. Following the construction of the reduced basis these $a_{p, n, m}$ are stored, and for each new problem the evaluation of the stiffness matrix is done, online, in $P \times N^{2}$ operations, and solved in $\mathcal{O}\left(\left(\operatorname{dim} X_{N}\right)^{3}\right)$ operations. These numbers are coherent with the rapid evaluation of the reduced basis method.

The hypothesis of affine parametric dependency is rather restrictive, and has to be generalized. In the case of quadratic or cubic dependency, the generalization is quite straightforward but even for linear problems such as Laplace or Stokes, when the geometry is the parameter, this is rarely the case and another approach has to be designed. In order to get a better understanding of the method, let us first assume that we want to compute $d_{\Omega}\left(\zeta_{i}, \zeta_{j}\right)$, defined as

$$
d_{\Omega}(u, v)=\int_{\Omega} u v d A=\int_{\hat{\Omega}} u v J_{\Phi} d \hat{A}
$$

where $J_{\Phi}$ is the Jacobian determinant of the transformation that maps $\hat{\Omega}$ onto $\Omega$ (we assume momentarily that there is no domain decomposition in order to make the presentation less cumbersome). There is no reason in the general case that $J_{\Phi}$ will be affine, and thus the previous approach will not work. It is nevertheless likely that there 
exists a sequence of well chosen transformations $\Phi_{1}^{*}, \ldots, \Phi_{M}^{*}, \ldots$ such that $J_{\Phi}$ may be well approximated by an expansion $J_{\Phi} \simeq \sum_{j=1}^{M} \beta_{j} J_{\Phi_{j}^{*}}$. An approximation of $d_{\Omega}\left(\zeta_{i}, \zeta_{j}\right)$ will then be given by

$$
d_{\Omega}\left(\zeta_{i}, \zeta_{j}\right) \simeq \sum_{j=1}^{M} \beta_{j} \int_{\hat{\Omega}} \hat{\zeta}_{i} \hat{\zeta}_{j} J_{\Phi_{j}^{*}} d \hat{A}
$$

and again, the contributions $\int_{\hat{\Omega}} \hat{\zeta}_{i} \hat{\zeta}_{j} J_{\Phi_{j}^{*}} d \hat{A}$ will be precomputed offline. We do not elaborate here on how the $\Phi_{j}^{*}$ are selected, we shall discuss this in more generality latter. What we want to address is the evaluation of the coefficients $\beta_{j}=\beta_{j}(\Omega)$ in the approximation of $J_{\Phi}$ above. The idea is to use an interpolation procedure as is explained in [12]. Let $\mathbf{x}_{1}$ be the point where $\left|J_{\Phi_{1}^{*}}\right|$ achieves its maximum value. Assuming then that $\mathbf{x}_{1}, \ldots, \mathbf{x}_{n}$ have been defined, and are such that the $n \times n$ matrix with entries $J_{\Phi_{k}^{*}}\left(\mathbf{x}_{\ell}\right), 1 \leq k, \ell \leq n$ is invertible, we define $\mathbf{x}_{n+1}$ as being the point where $r_{n+1}=\left|J_{\Phi_{n+1}^{*}}-\sum_{k=1}^{n} \gamma_{k} J_{\Phi_{k}^{*}}\right|$ achieves it maximum value. Here the scalars $\gamma_{k}$ are defined so that $r_{n+1}$ vanishes at any $\left(\mathbf{x}_{\ell}\right)$ for $\ell=1, \ldots, n$. This definition of the points $\mathbf{x}_{k}$ is possible as long the $\Phi_{j}$ are chosen such that the $J_{\Phi_{k}^{*}}$ are linearly independent (see [12]). The $\beta_{j}$ are then evaluated also through the interpolation process

$$
J_{\Phi}\left(\mathbf{x}_{\ell}\right)=\sum_{k=1}^{M} \beta_{k} J_{\Phi_{k}^{*}}\left(\mathbf{x}_{\ell}\right), \quad \forall 1 \leq \ell \leq M .
$$

We have not much theory confirming the very good results that we obtain. An indicator that allows us to be quite confident in the interpolation process is the fact that the Lebesgue constant attached to the previously built points is, in all the examples we have encountered, rather limited. (We remind that the Lebesgue constant is the maximum of the ratio between the interpolation error and the best fit error.)

The same process is now used when implementing the Navier-Stokes problem, where we can decide to compute the solution to the discrete version of (28) through an iterative process. Given a current approximation $\boldsymbol{u}_{N}^{p} \in V_{N}(\Omega)$, compute $\boldsymbol{u}_{N}^{p+1} \in V_{N}(\Omega)$ as the solution of

$$
a_{\Omega}\left(\boldsymbol{u}_{N}^{p+1}, \boldsymbol{v}\right)+c\left(\boldsymbol{u}_{N}^{p+1}, \boldsymbol{v} ; \boldsymbol{u}_{N}^{p}\right)=l_{\Omega}(\boldsymbol{v}), \quad \forall \boldsymbol{v} \in V_{N}(\Omega) .
$$

The evaluation of the stiffness matrix involves now the computation of $c\left(\zeta_{i}, \zeta_{j} ; \boldsymbol{u}_{N}^{p}\right)$, not only for each new geometry, but also at each iteration. It is an easy matter to realize that

$$
c\left(\zeta_{i}, \zeta_{j} ; \boldsymbol{u}_{N}^{p}\right)=\int_{\hat{\Omega}} J_{\Phi}\left[\boldsymbol{u}_{N}^{p}\right]^{t} \mathcal{J}_{\Phi}^{-1} \nabla \zeta_{i} \zeta_{j} d \hat{A}
$$

The online approximation of $J_{\Phi}\left[\boldsymbol{u}_{N}^{p}\right]^{t} \mathcal{J}_{\Phi}^{-1}$ is done through interpolation on appropriate collocation points based on a set of functions $J_{\Phi_{j}^{*}}\left[\boldsymbol{u}_{N}\right]^{t}\left(\Phi_{j}^{*}\right) \mathcal{J}_{\Phi_{j}^{*}}^{-1}, 1 \leq j \leq M$, where $\boldsymbol{u}_{N}\left(\Phi_{j}^{*}\right)$ is the converged and previously offline computed solution associated with the geometry $\Phi_{j}^{*}$. The construction of the updated part of the stiffness matrix is thus performed online in $\mathcal{O}\left(M N^{2}\right)$ operation, first by evaluating the $\beta_{k}$ such that $J_{\Phi}\left[\boldsymbol{u}_{N}^{p}\right]^{t} \mathcal{J}_{\Phi}^{-1} \simeq \sum_{k=1}^{M} \beta_{k} J_{\Phi_{k}^{*}}\left[\boldsymbol{u}_{N}\right]^{t}\left(\Phi_{k}^{*}\right) \mathcal{J}_{\Phi_{k}^{*}}^{-1}$, then by approximating $c\left(\zeta_{i}, \zeta_{j} ; \boldsymbol{u}_{N}^{p}\right)$ by

$$
c\left(\zeta_{i}, \zeta_{j} ; \boldsymbol{u}_{N}^{p}\right) \simeq \sum_{k=1}^{M} \beta_{k} \int_{\hat{\Omega}} J_{\Phi_{k}^{*}}\left[\boldsymbol{u}_{N}\right]^{t}\left(\Phi_{k}^{*}\right) \mathcal{J}_{\Phi_{k}^{*}}^{-1} \nabla \zeta_{i} \zeta_{j} d \hat{A} .
$$




\subsection{Transfinite mappings}

One major ingredient of the reduced basis element method is the design of the mapping between the reference domain and the current instantiation of the subdomain. This design has to be efficient, and the resulting mapping has to be regular enough. There is a large flexibility in the definition of the different possible mappings, but they should all be one-to-one and map the boundaries of the computational domain onto the boundaries of the physical domain. When the domain of reference is a square, a standard way of defining a mapping is the Gordon-Hall transfinite interpolation approach; see [10].

The idea behind transfinite interpolation is to construct the image of the interior points of the physical domain as linear combinations of the image of the points on the boundaries. On the reference domain, $\hat{\Omega}=(-1,1)^{2}$, we construct one-dimensional weight functions $\phi_{i}(r)$, such that for $r_{0}=-1$ and $r_{1}=1$ we get

$$
\phi_{i}\left(r_{j}\right)=\delta_{i j}, 0 \leq i, j \leq 1 .
$$

The weight functions may be linear, but this is not a necessity. We may also use different weight functions in different spatial directions.

We assume that a representation of the boundaries of the physical domain is given with respect to the reference variables $(\xi, \eta)$ by a bijective map. Each boundary will be the function of one variable, and we define the horizontal boundaries $\boldsymbol{x}(\xi,-1)$ and $\boldsymbol{x}(\xi, 1)$, and the vertical boundaries $\boldsymbol{x}(-1, \eta)$ and $\boldsymbol{x}(1, \eta)$, where $\boldsymbol{x}=(x, y)$.

The transfinite mapping is then defined as

$$
\begin{gathered}
\Phi(\xi, \eta)=\quad \phi_{0}(\xi) \boldsymbol{x}(-1, \eta)+\phi_{1}(\xi) \boldsymbol{x}(1, \eta)+\phi_{0}(\eta) \boldsymbol{x}(\xi,-1)+\phi_{1}(\eta) \boldsymbol{x}(\xi, 1) \\
-\sum_{i=0}^{1} \sum_{j=0}^{1} \phi_{i}(\xi) \phi_{j}(\eta) \boldsymbol{x}\left(r_{i}, r_{j}\right) .
\end{gathered}
$$

The mapping (34) will preserve the boundaries of the physical domain, and the interior points are determined via a linear transformation of the grid points defined on the reference domain.

When the reference domain $\hat{\Omega}$ is more complex, one way of working is to decompose it into a non-overlapping union of quadrilateral subdomains, do the same for the deformed domain $\Omega$ and define the mapping from $\hat{\Omega}$ onto $\Omega$ piecewise. The resulting mapping is generally continuous, piecewise regular but globally it is rarely $\mathcal{C}^{1}$. This is a redhibitory drawback for the use of the Piola transformation when dealing with the Stokes problem.

In order to improve the regularity of the mapping, we generalize (34) as follows. We assume $\hat{\Omega}$ and $\Omega$ are curved polygons with the same number of curved edges, say $n$. Let $\Gamma_{i}$ (resp. $\hat{\Gamma}_{i}$ ) denote each edge of $\Omega$ (resp. $\hat{\Omega}$ ) ranked in a clockwise manner and such that $\Gamma_{n+1}=\Gamma_{1}$. Let $\boldsymbol{x}_{i}$ (resp. $\hat{\boldsymbol{x}}_{i}$ ) denote the vertex between $\Gamma_{i}$ and $\Gamma_{i+1}$ (resp. $\hat{\Gamma}_{i}$ and $\left.\hat{\Gamma}_{i+1}\right)$. We assume that each edge is parametrized by a one to one mapping $\psi_{i}$ from ]0,1[ onto $\Gamma_{i}$ so that $\psi_{i}(1)=\boldsymbol{x}_{i}$ (with obvious extension for the reference domain). We assume also that we are given projection operators $\pi_{i}$ from $\hat{\Omega}$ onto $[0,1]$ that associate with any point over $\hat{\Gamma}_{i+1}$ the value 1 , with any point over $\hat{\Gamma}_{i-1}$ the value 0 and any point $\boldsymbol{x}$ over $\hat{\Gamma}_{i}$ the value $\hat{\psi}_{i}^{-1}(\boldsymbol{x})$. Finally, we introduce the weight functions $\varphi_{i}$ with values in $[0,1]$ that, similarly as in the original formulation satisfy $\varphi_{i}(\boldsymbol{x})=1$ over $\hat{\Gamma}_{i}$ and $\varphi_{i}(\boldsymbol{x})=0$ over any $\hat{\Gamma}_{j}$ with $j \neq i-1, i, i+1$. Then the mapping

$$
\Phi(\xi, \eta)=\sum_{i=1}^{n}\left\{\varphi_{i}(\xi, \eta) \psi_{i}\left[\pi_{i}(\xi, \eta)\right]-\varphi_{i}\left[\pi_{i+1}(\xi, \eta)\right] \varphi_{i+1}\left[\pi_{i}(\xi, \eta)\right] \boldsymbol{x}_{i}\right\}
$$


preserves the boundary of the domains. Under mild assumptions over the $\phi$ 's, it maps $\hat{\Omega}$ onto $\Omega$. We refer to [14] for more about this strategy.

\subsection{A posteriori error estimation}

The reduced basis methods are known for rapid convergence rates. For application to realistic complex problem you are not interested in showing nice convergence plots; you are interested in getting the answer to your problem at a minimal cost. In addition, you want the result to be reliable. The number of elements in the reduced basis to be used for a given accuracy depends on the problem and the only way to get a hint whether you have used a rich enough basis set, is to refer to a posteriori error estimations.

Furthermore, in most cases it is not so much the solution of the PDE that is interesting; it is most often some outputs that can be computed from the knowledge of the solution. These outputs of interest are regular functionals evaluated over the solution. Let us consider the Stokes problem (14). For some specified output of interest, $s(\boldsymbol{u})$, we are thus interested in providing, after the solution $\boldsymbol{u}_{N}$ has been found, a computable lower bound $s^{-}\left(\boldsymbol{u}_{N}\right)$ and a computable upper bound $s^{+}\left(\boldsymbol{u}_{N}\right)$ such that

$$
s^{-}\left(\boldsymbol{u}_{N}\right) \leq s(\boldsymbol{u}) \leq s^{+}\left(\boldsymbol{u}_{N}\right) .
$$

In this work, we focus on compliant output, i.e.

$$
s(\boldsymbol{u})=l_{\Omega}(\boldsymbol{u}) .
$$

and also, for the sake of simplification, on the mono-domain case.

We will follow the theory developed in [24] for operators which are continuous, coercive, symmetric and affine in terms of the parameter in a similar way as has been done in [28] for the steady Stokes problem with more standard parameter dependencies. The steady Stokes operator is symmetric and continuous, but not coercive, and due to the geometric dependency it is not affine either.

We introduce the diffusion operator

$$
\hat{a}(\boldsymbol{v}, \boldsymbol{w})=\int_{\hat{\Omega}} g(\Phi) \hat{\nabla} \hat{\boldsymbol{v}} \cdot \hat{\nabla} \hat{\boldsymbol{w}} d \hat{\Omega}
$$

on the reference domain, where $g(\Phi)$ is a geometry dependent positive function. The reconstructed error $\hat{\boldsymbol{e}} \in \hat{X}(\Omega)$ is then defined as the field that for some $g(\Phi)$ satisfies

$$
\hat{a}(\boldsymbol{e}, \boldsymbol{v})=l(\boldsymbol{v})-a\left(\boldsymbol{u}_{N}, \boldsymbol{v}\right)-b\left(\boldsymbol{v}, p_{N}\right) \quad \forall \hat{\boldsymbol{v}} \in \hat{X}(\Omega) .
$$

The operator $g(\Phi)$ is chosen such that

$$
\alpha_{0}\|\boldsymbol{v}\|_{X}^{2} \leq \hat{a}(\boldsymbol{v}, \boldsymbol{v}) \leq a(\boldsymbol{v}, \boldsymbol{v}) \quad \forall \boldsymbol{v} \in X(\Omega),
$$

for some positive real constant $\alpha_{0}$. For this reconstructed error we claim that

$$
\begin{gathered}
s^{-}\left(\boldsymbol{u}_{N}\right)=l\left(\boldsymbol{u}_{N}\right), \text { and } \\
s^{+}\left(\boldsymbol{u}_{N}\right)=l\left(\boldsymbol{u}_{N}\right)+\hat{a}(\boldsymbol{e}, \boldsymbol{e}),
\end{gathered}
$$

are lower and upper bounds for $s(\boldsymbol{u})$.

Before we prove (40) and (41), we put $\boldsymbol{v}=\boldsymbol{u}_{N}$ in (14) and (26) to see that

$$
a\left(\boldsymbol{u}_{N}, \boldsymbol{u}-\boldsymbol{u}_{N}\right)+b\left(\boldsymbol{u}_{N}, p\right)=0 .
$$


The last term is zero and thus (42) reduces to

$$
a\left(\boldsymbol{u}_{N}, \boldsymbol{u}-\boldsymbol{u}_{N}\right)=0 .
$$

For the lower bound we get

$$
\begin{aligned}
s^{-}\left(\boldsymbol{u}_{N}\right) & =s(\boldsymbol{u})+l\left(\boldsymbol{u}_{N}-\boldsymbol{u}\right) \\
& =s(\boldsymbol{u})+a\left(\boldsymbol{u}, \boldsymbol{u}_{N}-\boldsymbol{u}\right)+b\left(\boldsymbol{u}_{N}-\boldsymbol{u}, p\right) \\
& =s(\boldsymbol{u})+a\left(\boldsymbol{u}, \boldsymbol{u}_{N}-\boldsymbol{u}\right)+a\left(\boldsymbol{u}_{N}, \boldsymbol{u}-\boldsymbol{u}_{N}\right) \\
& =s(\boldsymbol{u})+a\left(\boldsymbol{u}-\boldsymbol{u}_{N}, \boldsymbol{u}_{N}-\boldsymbol{u}\right) .
\end{aligned}
$$

And we have the desired relationship

$$
s^{-}\left(\boldsymbol{u}_{N}\right) \leq s(\boldsymbol{u}),
$$

independent of $g(\Phi)$.

For the upper bound we denote the error on the deformed domain by $\boldsymbol{e}^{u}=\boldsymbol{u}-\boldsymbol{u}_{N}$, and find that

$$
\begin{aligned}
2 \hat{a}\left(\boldsymbol{e}, \boldsymbol{e}^{u}\right)= & l\left(\boldsymbol{u}-\boldsymbol{u}_{N}\right)+l\left(\boldsymbol{u}-\boldsymbol{u}_{N}\right) \\
& -2 a\left(\boldsymbol{u}_{N}, \boldsymbol{u}-\boldsymbol{u}_{N}\right) \\
= & l\left(\boldsymbol{u}-\boldsymbol{u}_{N}\right)+a\left(\boldsymbol{u}, \boldsymbol{u}-\boldsymbol{u}_{N}\right) \\
& +b\left(\boldsymbol{u}-\boldsymbol{u}_{N}, p\right)-2 a\left(\boldsymbol{u}_{N}, \boldsymbol{u}-\boldsymbol{u}_{N}\right) \\
= & l\left(\boldsymbol{u}-\boldsymbol{u}_{N}\right)+a\left(\boldsymbol{u}-\boldsymbol{u}_{N}, \boldsymbol{u}-\boldsymbol{u}_{N}\right) \\
& -a\left(\boldsymbol{u}_{N}, \boldsymbol{u}-\boldsymbol{u}_{N}\right) \\
= & l\left(\boldsymbol{u}-\boldsymbol{u}_{N}\right)+a\left(\boldsymbol{e}^{u}, \boldsymbol{e}^{u}\right) .
\end{aligned}
$$

To prove that (41) is an upper bound we now use (46) to get

$$
\begin{aligned}
s^{+}\left(\boldsymbol{u}_{N}\right)= & l\left(\boldsymbol{u}_{N}\right)+\hat{a}(\boldsymbol{e}, \boldsymbol{e}) \\
= & l\left(\boldsymbol{u}_{N}\right)+\hat{a}(\boldsymbol{e}, \boldsymbol{e}) \\
& -2 \hat{a}\left(\boldsymbol{e}, \boldsymbol{e}^{u}\right)+l\left(\boldsymbol{u}-\boldsymbol{u}_{N}\right)+a\left(\boldsymbol{e}^{u}, \boldsymbol{e}^{u}\right) \\
& +\hat{a}\left(\boldsymbol{e}^{u}, \boldsymbol{e}^{u}\right)-\hat{a}\left(\boldsymbol{e}^{u}, \boldsymbol{e}^{u}\right) \\
= & l(\boldsymbol{u})+\hat{a}\left(\boldsymbol{e}-\boldsymbol{e}^{u}, \boldsymbol{e}-\boldsymbol{e}^{u}\right) \\
& +a\left(\boldsymbol{e}^{u}, \boldsymbol{e}^{u}\right)-\hat{a}\left(\boldsymbol{e}^{u}, \boldsymbol{e}^{u}\right) \\
\geq & s(\boldsymbol{u}),
\end{aligned}
$$

where the inequality is due to $(39)$ and the coercivity of $\hat{a}(\cdot, \cdot)$.

It now remains to find a positive function $g(\Phi)$, such that

$$
a(\boldsymbol{v}, \boldsymbol{v}) \geq \hat{a}(\boldsymbol{v}, \boldsymbol{v}) \quad \forall \boldsymbol{v} \in X(\Omega) .
$$

For a constant $g(\Phi)=\lambda$, we can use the theory of [24] to see that $\lambda$ should be chosen as large as possible without violating (48). This largest constant may be found, as in [18], by computing the smallest eigenvalue of the generalized symmetric eigenvalue problem

$$
a(\boldsymbol{v}, \boldsymbol{v})=\lambda \int_{\hat{\Omega}} \hat{\nabla}(\boldsymbol{v} \circ \Phi) \cdot \hat{\nabla}(\boldsymbol{v} \circ \Phi) d \hat{\Omega} .
$$

We tried this approach also for the current problem, and used an inverse Rayleigh quotient iteration to estimate $\lambda$, but the resulting upper bound gap proved much too conservative.

To get a better estimate we consider the Jacobian, $\mathcal{J}(\Phi)$, of the mapping from $\hat{\Omega}$ to $\Omega$. We start with the left hand side of (48), and use the fact that

$$
\nabla=\mathcal{J}^{-T} \hat{\nabla}
$$


to rewrite (15) mapped back on the reference domain

$$
\begin{aligned}
a(\boldsymbol{v}, \boldsymbol{v}) & =\int_{\hat{\Omega}}(\hat{\nabla}[\boldsymbol{v} \circ \Phi])^{T} \mathcal{J}^{-1} \mathcal{J}^{-T}(\hat{\nabla}[\boldsymbol{v} \circ \Phi])|J| d \hat{\Omega} \\
& =\int_{\hat{\Omega}} \boldsymbol{w}^{T} G \boldsymbol{w} d \hat{\Omega},
\end{aligned}
$$

where $\boldsymbol{w}=\hat{\nabla}[\boldsymbol{v} \circ \Phi]$, and $G=G(\Phi)=\mathcal{J}^{T} \mathcal{J}|J|$. At each point $\hat{\mathbf{x}} \in \Omega$ we diagonalize the 2 symmetric positive-definite matrix $G$, that is, we write $G(\Phi(\hat{\mathbf{x}}))=Q^{T} \Lambda Q$, where $Q$ consists of the orthonormal eigenvectors of $G$. If we (at each point $\hat{\mathbf{x}} \in \hat{\Omega}$ ) replace the two diagonal elements of $\Lambda$ with the smallest one $\Lambda_{\min }$, we get

$$
\int_{\hat{\Omega}} \boldsymbol{w}^{T} G \boldsymbol{w} d \hat{\Omega} \geq \int_{\hat{\Omega}} \Lambda_{\min }(Q \boldsymbol{w})^{T} Q \boldsymbol{w} d \hat{\Omega}
$$

Since $Q$ consists of the orthonormal eigenvectors, the last expression is equivalent to $\int_{\hat{\Omega}} \Lambda_{\min } \boldsymbol{w}^{T} \boldsymbol{w} d \hat{\Omega}$, and we end up with

$$
a(\boldsymbol{v}, \boldsymbol{v}) \geq \int_{\hat{\Omega}} \Lambda_{\min }(\hat{\nabla}[\boldsymbol{v} \circ \Phi]) \cdot(\hat{\nabla}[\boldsymbol{v} \circ \Phi]) d \hat{\Omega} .
$$

This is just (37) with $g(\Phi)=\Lambda_{\min }(\Phi)$, and thus (48) is satisfied.

If we replace $\Lambda_{\min }(\Phi)$ by $\bar{\Lambda}_{\text {min }}=\min _{\hat{\mathbf{x}} \in \hat{\Omega}} \Lambda_{\min }(\Phi)$, we may put $g(\Phi)$ outside the integral and apply the theory of [24]. This will produce a more conservative upper bound, but the calculation of (41) can be split in an off-line/online procedure without applying the methodology of subsection 4.1 .

To illustrate numerically the results on the output bounds, we will use the domain $\Omega=\Phi(\hat{\Omega})$ defined in Figure 8 .
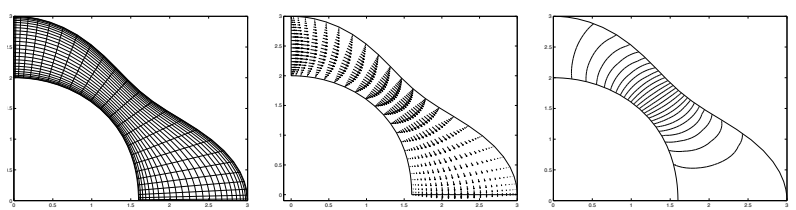

Figure 8: The domain $\Omega$ with velocity and pressure solution

The results for the previously defined deformed geometry are presented in Table 1. We see that the upper bound gap is relatively large compared to the lower bound gap. In the future, a different method to find an improved estimate of $g(\Phi)$ is desirable to reduce the upper bound gap.

We end this subsection by explaining how these a posteriori estimates can be used in order to select the basis solutions that are worth keeping to represent in an optimal way the set of all solutions. The strategy is based on a greedy algorithm in which the first parameter is selected at random, or at least so that the output is not zero. Then assuming that $m$ basis functions are selected, the selection of the $m+1$ parameter corresponds to the argmax of the a posteriori error on the output based on the approximation with the discrete space spanned with the $m$ selected basis functions.

By choosing this argmax, we are indeed sure that the corresponding solution is quite far from the vectorial space spanned by the $m$ first basis functions. 


\begin{tabular}{|c|c|c|c|}
\hline$N$ & $s(\boldsymbol{u})-s^{-}\left(\boldsymbol{u}_{N}\right)$ & $s^{+}\left(\boldsymbol{u}_{N}\right)-s(\boldsymbol{u})$ & $s_{2}^{+}\left(\boldsymbol{u}_{N}\right)-s(\boldsymbol{u})$ \\
\hline 1 & $2.82 \cdot 10^{-4}$ & $5.28 \cdot 10^{-2}$ & $1.01 \cdot 10^{-1}$ \\
2 & $1.87 \cdot 10^{-4}$ & $9.86 \cdot 10^{-2}$ & $1.85 \cdot 10^{-1}$ \\
3 & $1.35 \cdot 10^{-4}$ & $9.74 \cdot 10^{-2}$ & $1.90 \cdot 10^{-1}$ \\
4 & $1.32 \cdot 10^{-4}$ & $9.70 \cdot 10^{-2}$ & $1.86 \cdot 10^{-1}$ \\
5 & $7.67 \cdot 10^{-5}$ & $3.02 \cdot 10^{-2}$ & $4.52 \cdot 10^{-2}$ \\
6 & $7.44 \cdot 10^{-5}$ & $6.86 \cdot 10^{-3}$ & $1.20 \cdot 10^{-2}$ \\
7 & $1.04 \cdot 10^{-5}$ & $1.56 \cdot 10^{-3}$ & $2.60 \cdot 10^{-3}$ \\
8 & $7.03 \cdot 10^{-6}$ & $2.62 \cdot 10^{-3}$ & $4.32 \cdot 10^{-3}$ \\
9 & $7.02 \cdot 10^{-6}$ & $1.61 \cdot 10^{-3}$ & $2.39 \cdot 10^{-3}$ \\
10 & $4.24 \cdot 10^{-6}$ & $6.10 \cdot 10^{-4}$ & $1.02 \cdot 10^{-3}$ \\
11 & $4.16 \cdot 10^{-6}$ & $6.21 \cdot 10^{-4}$ & $1.05 \cdot 10^{-3}$ \\
12 & $3.15 \cdot 10^{-6}$ & $6.16 \cdot 10^{-4}$ & $9.68 \cdot 10^{-4}$ \\
13 & $2.82 \cdot 10^{-6}$ & $4.63 \cdot 10^{-4}$ & $7.50 \cdot 10^{-4}$ \\
14 & $1.94 \cdot 10^{-6}$ & $4.32 \cdot 10^{-4}$ & $6.97 \cdot 10^{-4}$ \\
15 & $1.94 \cdot 10^{-6}$ & $3.82 \cdot 10^{-4}$ & $6.71 \cdot 10^{-4}$ \\
\hline
\end{tabular}

Table 1: Convergence of the lower and the upper bound gaps. Here, $s^{+}$corresponds to the variable $g(\Phi)=\Lambda_{\min }(\Phi)$, while $s_{2}^{+}$corresponds to the constant $g(\Phi)=\bar{\Lambda}_{\min }(\Phi)$.

\section{$5 \quad$ Numerical results}

\subsection{The Stokes problem}

For the hierarchical flow system presented in Figure 4 left, we construct basis functions for the pipe and bifurcation blocks separately. For the pipe we vary the deformation of the walled part of the boundary, and also the orientation of the outflow boundary relative to the inflow boundary. For the bifurcation we vary the opening angle of the two legs of the bifurcation, and the relative length of the two legs. The basis functions are computed as described above (truncating a multidomain solution, and applying the greedy algorithm). Using these basis functions for the pipe block and the three bifurcation blocks, we get the results presented in Table 2 for the steady Stokes problem.

We have used the same basis functions to approximate the solution of the bypass configuration shown in Figure 9. The results for the steady Stokes problem for this system are presented in Table 3 .

\subsection{The Navier Stokes problem}

The experiment on the steady Navier-Stokes problem is done on a monodomain pipe. The basis functions are found on a deformed quarter annulus by varying the deformation of the inner curved boundary. To solve the steady Navier-Stokes problem, we consider the corresponding time-dependent problem, and iterate in time until we reach a steady state solution. In this way we find seven basis function, and a reference solution. The convergence of the reduced basis method is presented in Table 4. 


\begin{tabular}{|c|c|c|c|c|}
\hline$N$ & $N_{1}$ & $N_{2}$ & $\left|\boldsymbol{u}_{N}-\boldsymbol{u}\right|_{H^{1}}$ & $\left\|p_{N}-p\right\|_{L^{2}}$ \\
\hline 36 & 9 & 9 & $2.6 \cdot 10^{-3}$ & $4.0 \cdot 10^{-1}$ \\
44 & 11 & 11 & $1.7 \cdot 10^{-3}$ & $6.6 \cdot 10^{-2}$ \\
52 & 13 & 13 & $1.2 \cdot 10^{-3}$ & $4.9 \cdot 10^{-2}$ \\
65 & 15 & 15 & $1.1 \cdot 10^{-3}$ & $3.7 \cdot 10^{-2}$ \\
105 & 15 & 30 & $4.2 \cdot 10^{-4}$ & $6.3 \cdot 10^{-3}$ \\
\hline
\end{tabular}

Table 2: The error in the reduced basis steady Stokes solution on a multi-block system corresponding to Figure 4 left. Here, $N=N_{1}+3 N_{2}$ is the total number of degrees-of-freedom in the reduced basis spaces $X_{N}^{0}, X_{N}^{e}$, and $M_{N}, N_{1}$ is the number of basis geometries used to generate the basis functions on the pipe block, and $N_{2}$ is the number of basis functions used on the bifurcation blocks.

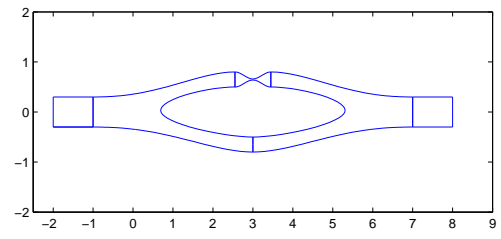

Figure 9: A bypass. 


\begin{tabular}{|c|c|c|c|c|}
\hline$N$ & $N_{1}$ & $N_{2}$ & $\left|\boldsymbol{u}_{N}-\boldsymbol{u}\right|_{H^{1}}$ & $\left\|p_{N}-p\right\|_{L^{2}}$ \\
\hline 45 & 9 & 9 & $9.3 \cdot 10^{-3}$ & $3.3 \cdot 10$ \\
55 & 11 & 11 & $3.1 \cdot 10^{-3}$ & $5.3 \cdot 10^{-1}$ \\
65 & 13 & 13 & $2.3 \cdot 10^{-3}$ & $9.0 \cdot 10^{-2}$ \\
75 & 15 & 15 & $1.4 \cdot 10^{-3}$ & $5.3 \cdot 10^{-2}$ \\
105 & 15 & 30 & $5.4 \cdot 10^{-4}$ & $3.0 \cdot 10^{-2}$ \\
\hline
\end{tabular}

Table 3: The error in the reduced basis steady Stokes solution on a multi-block bypass with three pipe blocks and two bifurcation blocks. Here, $N=3 N_{1}+2 N_{2}$ is the total number of degrees-of-freedom in each of the reduced basis spaces $X_{N}^{0}, X_{N}^{e}$, and $M_{N}, N_{1}$ is the number of basis geometries used to generate the basis functions on the pipe block, and $N_{2}$ is the number of basis functions used on the bifurcation blocks.

\begin{tabular}{|c|c|c|c|c|}
\hline$N / 2$ & $\left|u_{N}-u\right|_{H^{1}}$ & $\| p_{N}-\left.p\right|_{L^{2}}$ & $s_{N}^{+}-s$ & $s-s_{N}^{-}$ \\
\hline 1 & $1.5 \cdot 10^{-1}$ & $1.2 \cdot 10^{-1}$ & 1.1 & $3.9 \cdot 10^{-3}$ \\
2 & $1.7 \cdot 10^{-2}$ & $1.1 \cdot 10^{-2}$ & $1.5 \cdot 10^{-2}$ & $1.0 \cdot 10^{-3}$ \\
3 & $2.0 \cdot 10^{-3}$ & $8.8 \cdot 10^{-4}$ & $3.8 \cdot 10^{-4}$ & $2.6 \cdot 10^{-4}$ \\
4 & $1.1 \cdot 10^{-4}$ & $2.5 \cdot 10^{-5}$ & $1.0 \cdot 10^{-5}$ & $5.8 \cdot 10^{-6}$ \\
5 & $5.3 \cdot 10^{-5}$ & $3.6 \cdot 10^{-6}$ & $2.5 \cdot 10^{-6}$ & $1.5 \cdot 10^{-6}$ \\
6 & $5.1 \cdot 10^{-5}$ & $3.4 \cdot 10^{-6}$ & $1.3 \cdot 10^{-6}$ & $1.4 \cdot 10^{-6}$ \\
7 & $3.9 \cdot 10^{-5}$ & $2.1 \cdot 10^{-6}$ & $2.2 \cdot 10^{-7}$ & $4.2 \cdot 10^{-7}$ \\
\hline
\end{tabular}

Table 4: Results when we use offline/online decoupling. The error in the reduced basis solution of the Navier-Stokes problem when the stopping criterion for the truth solution is $10^{-10}$. 
Acknowledgement: This project on the development of the reduced basis element method for fluid flow has greatly benefitted from the longstanding collaboration on the reduced basis methods with Professor Anthony Patera of MIT and his team. This work was supported by the ACI-NIM "LE-POUMON-VOUS-DIS-JE", and by the Research Council of Norway through the BeMatA programme under contract 147044/431.

\section{References}

[1] B.O. Almroth, P. Stern, F.A. Brogan - Automatic choice of global shape functions in structural analysis. AIAA Journal, 16 (1978) 525528.

[2] L. Baffico, C. Grandmont, Y. Maday, and A. Osses - Homogenization of an elastic media with gaseous bubbles, in preparation.

[3] F. Brezzi and M. Fortin - Mixed and Hybrid Finite Element Methods. Springer Verlag, 1991.

[4] M. Briane, Y. Maday, and F. Madigou - Homogenization of a two-dimensional fractal conductivity, in preparation.

[5] C.Fetita, S.M, D.Perchet, F.Prêteux, M.Thiriet, and L.Vial - An image-based computational model of oscillatory flow in the proximal part of tracheobronchial trees, Computer Methods in Biomechanics and Biomedical Engineering, 8(4),279293, (2005).

[6] JP Fink and WC Rheinboldt - On the error behavior of the reduced basis technique for nonlinear finite element approximations. Zeitschrift fr Angewandte Mathematik und Mechanik, 63(1) (1983) 2128.

[7] R.L. Fox and H. Miura - An approximate analysis technique for design calculations. AIAA Journal, 9(1) (1971) 177179.

[8] M B Giles and E Süli - Adjoint methods for PDEs: a posteriori error analysis and postprocessing by duality. Acta Numerica, Vol. 11, 145-236, Cambridge University Press, 2002.

[9] V. Girault, P.A. Raviart — Finite element methods for Navier-Stokes equations: Theory and algorithms, (Springer Series in Computational Mathematics. Volume 5), Berlin and New York, Springer-Verlag (1986)

[10] W. Gordon and C. Hall — Transfinite element methods: blending-function interpolation over arbitrary curved element domains, Numer. Math. (21), 1973/74, pp 109-129.

[11] C. Grandmont, Y. Maday, and B. Maury - A multiscale/multimodel approach of the respiration tree, in New Trends in Continuum Mechanics - M. MihailescuSuliciu, Ed. - Theta Foundation, Bucharest, Romania (2005).

[12] M. A. Grepl, Y. Maday, N. C. Nguyen, and A. T. Patera - Efficient reduced basis treatment of non-affine and nonlinear partial differential equations, submitted to $\mathrm{M}^{2} \mathrm{AN}(2006)$.

[13] A. E. Løvgren, Y. Maday, and E. M. Rønquist - A reduced basis element method for the steady Stokes problem, to appear in $\mathrm{M}^{2} \mathrm{AN}$.

[14] A. E. Løvgren, Y. Maday, and E. M. Rønquist - in progress. 
[15] L. Machiels, Y. Maday, I.B Oliveira, A.T. Patera, and D.V. Rovas - Output bounds for reduced-basis approximations of symmetric positive definite eigenvalue problems, CR Acad Sci Paris Series I 331:(2000) 153158.

[16] Y. Maday, A. T. Patera, and G. Turinici - A Priori Convergence Theory for Reduced-Basis Approximations of Single-Parameter Elliptic Partial Differential Equations, J. Sci. Comput., 17 (2002), no. 1-4, 437-446.

[17] Y. Maday, L. Machiels, A.T. Patera, and D.V. Rovas — Blackbox reduced-basis output bound methods for shape optimization, in Proceedings 12th International Domain Decomposition Conference, Chiba, Japan (2000) 429436.

[18] Y. Maday and E. M. Rønquist - The reduced-basis element method: Application to a thermal fin problem. SIAM Journal on Scientific Computing, 2004.

[19] AK Noor and JM Peters - Reduced basis technique for nonlinear analysis of structures. AIAA Journal, 18(4) (1980) 455462.

[20] V. Milisic and A. Quarteroni. Analysis of lumped parameter models for blood flow simulations and their relation with 1D models, to appear in $\mathrm{M}^{2} \mathrm{AN}, 2004$.

[21] F. Murat and J. Simon, Sur le Controle par un Domaine Geometrique, Publication of the Laboratory of Numerical Analysis, University Paris VI, 1976.

[22] Pinkus, A. - n-Widths in Approximation Theory, Springer-Verlag, Berlin (1985).

[23] TA Porsching - Estimation of the error in the reduced basis method solution of nonlinear equations. Mathematics of Computation. 45(172) (1985) 487496.

[24] C. Prud'homme, D.V. Rovas, K . Veroy, L. Machiels, Y. Maday, A.T. Patera, G. Turinici - Reliable real-time solution of parametrized partial differential equations: Reduced-basis output bound methods, J Fluids Engineering, 124, (2002) $70-80$.

[25] C. Prud'homme, D. V. Rovas, K. Veroy, and A. T. Patera - A mathematical and computational framework for reliable real-time solution of parametrized partial differential equations. Programming. $\mathrm{M}^{2}$ AN Math. Model. Numer. Anal. 36 (2002), no. 5, 747-771.

[26] A. Quarteroni and A. Valli - Domain decomposition methods for partial differential equations, Numerical Mathematics and Scientific Computation, Oxford Science Publications, The Clarendon Press Oxford University Press, New York, (1999).

[27] P.A. Raviart and J.M. Thomas. - A mixed finite element method for 2-nd order elliptic problems. In I. Galligani and E. Magenes, editors, Mathematical Aspects of Finite Element Methods, Lecture Notes in Mathematics, Vol. 606. SpringerVerlag, 1977.

[28] D. V. Rovas. - Reduced-Basis Output Bound Methods for Parametrized Partial Differential Equations. PhD thesis, Massachusetts Institute of Technology, Cambridge, MA, October 2002.

[29] M. Thiriet - http://www-rocq.inria.fr/who/Marc.Thiriet/Vitesv/

[30] A. Toselli and O. Widlund - Domain decomposition methods-algorithms and theory, Springer Series in Computational Mathematics, 34, Springer-Verlag, Berlin, (2005).

[31] R. Verfurth - A Review of A Posteriori Error Estimation and Adaptive MeshRefinement Techniques. Wiley-Teubner, 1996. 